medRxiv preprint doi: https://doi.org/10.1101/2022.02.24.22271449; this version posted March 2, 2022. The copyright holder for this preprint (which was not certified by peer review) is the author/funder, who has granted medRxiv a license to display the preprint in perpetuity. It is made available under a CC-BY-NC-ND 4.0 International license.

\title{
Healthcare services access, use, and barriers among migrants in Europe: a systematic review
}

Petros Galanis, Assistant Professor, Clinical Epidemiology Laboratory, Faculty of Nursing, National and Kapodistrian University of Athens, Greece

Koureas Spyros, MSc, PhD (c), National and Kapodistrian University of Athens, Greece Olga Siskou, Assistant Professor, University of Piraeus, Greece

Olympia Konstantakopoulou, Economist, MSc, $\mathrm{PhD}$, Center for Health Services Management and Evaluation, National and Kapodistrian University of Athens, Greece

Georgios Angelopoulos, Economist, MSc, Center for Health Services Management and Evaluation, National and Kapodistrian University of Athens, Greece

Daphne Kaitelidou, Professor, Center for Health Services Management and Evaluation, National and Kapodistrian University of Athens, Greece

Corresponding author: Petros Galanis, Assistant Professor, Clinical Epidemiology Laboratory, Faculty of Nursing, National and Kapodistrian University of Athens, Greece, 123 Papadiamantopoulou street, GR-11527, Athens, Greece, Tel:+30210.7781044, email: pegalan@nurs.uoa.gr

Funding: National and Kapodistrian University of Athens and "Stavros Niarchos Foundation" 
medRxiv preprint doi: https://doi.org/10.1101/2022.02.24.22271449; this version posted March 2, 2022. The copyright holder for this preprint (which was not certified by peer review) is the author/funder, who has granted medRxiv a license to display the preprint in perpetuity.

\begin{abstract}
Background: The issue of migrants health and access to health services is dynamic and complex posing a challenge to health systems worldwide.

Aim: To investigate migrants' access to health services in European countries, the use of health services by migrants and the barriers encountered by migrants in the use of health services.
\end{abstract}

Material and methods: The search was conducted in January 2022 in five databases; PubMed, Medline, Web of science, Scopus and Cinahl. We used the following keywords: migrants, immigrants, use, access, utilization, healthcare services, services, needs, health, difficulties, barriers. The inclusion criteria were the following: (a) the studies investigated the access of migrants to health services, the use of health services by migrants and the barriers encountered by migrants in using health services. (b) migrants self-assessed access, use and barriers. (c) studies were conducted in European countries. (d) studies included adult migrants. (e) the language of articles was English.

Results: Sixty-five studies were met our inclusion criteria. among studies, $89.2 \%$ were quantitative and $11.8 \%$ were qualitative. All quantitative studies were cross-sectional. for data collection, $58.5 \%$ of studies used questionnaires and $30.8 \%$ used historical files. Also, personal interviews were performed in $9.2 \%$ of studies and focus groups in $1.5 \%$ of studies. in our studies, $73.8 \%$ of natives stated that they had better access to health services and used health services better than migrants, while $26.2 \%$ found that migrants stated that they had better access to health services and used health services better. The most common barriers were the following: inability to understand the language and communicate, lack of insurance, lack of information and knowledge, lack of family support, low educational level, short duration of stay in the country of migration, low income, lack of a family doctor and high costs.

Conclusions: Migrants face several barriers both in accessing and using health services in Europe. Intensive efforts are needed to increase migrants' knowledge, implement culturally sensitive interventions in migrant communities and better inform healthcare professionals so that they can approach migrants more effectively.

Keywords: migrants, Europe, access, use, barriers, healthcare, services 
medRxiv preprint doi: https://doi.org/10.1101/2022.02.24.22271449; this version posted March 2, 2022. The copyright holder for this preprint

\section{Introduction}

The high mobility of populations, the economic crisis and the significant increase in unemployment have defined the last decades, with the economic crisis affecting vulnerable groups, such as migrants. Thus, a particular aspect of health inequalities has emerged, which mainly concerns the barriers and difficulties encountered by migrants in accessing healthcare services, which has a direct impact on the quality of the services provided. The Americas is the continent with the largest number of migrants, with the number increasing from 47 million in 2000 to 60 million in 2012 (Loue \& Sajatovic, 2012). The situation is similar in Europe, with migrants making up around $9 \%$ of the total population (Loue \& Sajatovic, 2012). In fact, in Europe, 7-13\% of migrants do not have legal residence documents (Karl-Trummer et al., 2010).

Access to high quality health services is an extremely important issue for migrants, as they face unequal opportunities for treatment interventions (The Lancet Public Health, 2018). The growing migrant population is also reflected in the increasing need for health services. For this reason, a corresponding adjustment of health policies at international level is required to better provide health care for migrants (Macpherson et al., 2007). Indeed, in recent years the issue of migrants' health has expanded from providing health care to patients to health promotion and prevention. The World Health Organization plays an essential role in better addressing health issues related to migrants (World Health Organization, 2008).

The issue of migrants health and access to health services is dynamic and complex, involving different stages of migration such as the pre-migration period, the first years of migration and migrants after several years of residence in the host country (Lassetter \& Callister, 2009; Ullmann et al., 2011). Furthermore, this issue is also related to various social determinants that are not only related to the characteristics of migrants, e.g. gender, cultural diversity, experiences, etc., but also to the cultural environment of the host countries, e.g. the health care system, living conditions, moral, religious and cultural values, etc. (Malmusi et al., 2010; Spallek et al., 2011).

Migrants and their children are less often insured and use health services less often than natives (Abe-Kim et al., 2007; Alegría et al., 2006; Callahan et al., 2006; 
medRxiv preprint doi: https://doi.org/10.1101/2022.02.24.22271449; this version posted March 2, 2022. The copyright holder for this preprint (which was not certified by peer review) is the author/funder, who has granted medRxiv a license to display the preprint in perpetuity.

Cunningham, 2006; Guendelman et al., 2001; Huang et al., 2006; Jackson et al., 2007; Javier et al., 2007; Lasser et al., 2006; Yu et al., 2006). In addition, the cost of health care for migrants is lower, with the exception of the cost of emergency care for migrant children (Derose et al., 2009).

The aim of this systematic literature review was to investigate migrants' access to health services, the use of health services by migrants and the barriers encountered by migrants in the use of health services.

\section{Material and Methods}

The search was conducted in January 2022 in five databases; PubMed, Medline, Web of Science, Scopus and Cinahl. We used the following key-words: migrants, immigrants, use, access, utilization, healthcare services, services, needs, health, difficulties, barriers.

The inclusion criteria were the following: (a) the studies investigated the access of migrants to health services, the use of health services by migrants and the barriers encountered by migrants in using health services. (b) migrants self-assessed access, use and barriers. (c) studies were conducted in European countries. (d) studies included adult migrants. (e) the language of articles was English

Studies that included in the systematic literature review are presented in Table 1, while the flowchart is presented in Figure 1.

\section{Results}

Sixty-five studies were met our inclusion criteria. Countries that studies were conducted in our review were Spain, Italy, United Kingdom, Greece, Austria, Belgium, France, Netherlands, Wales, Portugal, Germany, Denmark, Switzerland, Norway, Sweden and Czech Republic.

Among studies, $89.2 \%$ were quantitative and $11.8 \%$ were qualitative. All quantitative studies were cross-sectional. In qualitative studies, the minimum sample size was 10 and the maximum number was 55, while in the quantitative studies, the minimum sample size was 74 and the maximum number was 3,739,244. 
medRxiv preprint doi: https://doi.org/10.1101/2022.02.24.22271449; this version posted March 2, 2022. The copyright holder for this preprint (which was not certified by peer review) is the author/funder, who has granted medRxiv a license to display the preprint in perpetuity.

It is made available under a CC-BY-NC-ND 4.0 International license .

For data collection, $58.5 \%$ of studies used questionnaires and $30.8 \%$ used historical files. Also, personal interviews were performed in $9.2 \%$ of studies and focus groups in $1.5 \%$ of studies.

Natives were also included in $73.8 \%$ of studies allowing comparisons with migrants. In these studies, $73.8 \%$ of natives stated that they had better access to health services and used health services better than migrants, while $26.2 \%$ found that migrants stated that they had better access to health services and used health services better.

Among studies, $18.5 \%$ investigated migrants' access to health services in general, while $81.5 \%$ investigated access to specific health services, such as primary health care services, general practitioner, emergency department, hospitalization, etc.

The barriers faced by migrants in accessing and using health services are shown in Table 2. The most common barriers were the following: inability to understand the language and communicate, lack of insurance, lack of information and knowledge, lack of family support, low educational level, short duration of stay in the country of migration, low income, lack of a family doctor and high costs.

The barriers that migrants face in accessing and using health services can be summarized in the following five broad categories: social and economic barriers, health system-related barriers, cultural barriers, knowledge-related barriers and personal barriers.

Social barriers included low educational attainment, lack of insurance, high costs, low income, frequent travel, limited social integration and inability to understand language and communication.

Barriers related to the health system included lack of a family doctor, limited understanding of doctors' instructions, long waiting time, difficulties in access, lack of translators and living in rural areas.

Cultural barriers included stigma, shame and modesty on the part of females and religious beliefs.

Knowledge-related barriers included lack of knowledge about how to access and navigate the health system, lack of knowledge about screening, perception that screening is unnecessary and self-perception of good health status. 
medRxiv preprint doi: https://doi.org/10.1101/2022.02.24.22271449; this version posted March 2, 2022. The copyright holder for this preprint (which was not certified by peer review) is the author/funder, who has granted medRxiv a license to display the preprint in perpetuity. It is made available under a CC-BY-NC-ND 4.0 International license .

Personal barriers include lack of family support, short length of stay in the country of migration, fear, lack of time, anxiety, lack of confidence and functional incapacity.

\section{Discussion}

According to our review, migrants face several barriers both in accessing and using health services.

Firstly, there are significant social and economic barriers that limit migrants' access to health services, as well as their use. More specifically, low social and economic level, as well as living in areas characterized by low income, is a determining factor that reduces the use of health services by migrants, especially those services related to screening, such as in the case of mammography (Ahmad et al., 2012; Donnelly et al., 2009). In many cases, migrants with low social and economic status are paid an hourly rate, resulting in the use of health services leading to lost time and thus reduced income (Donnelly et al., 2009; Meana et al., 2001). This is supported by the fact that moving to health services requires some costs. A typical example is women with a low level of education and limited social integration, who make very little use of screening services (Donnelly et al., 2009; Meana et al., 2001). It is noted that low educational and economic level also leads to a lack of knowledge and understanding which further limits the use of health services by migrants. It is clear that the high cost of some services acts as a deterrent to their use by migrants, as they are unable to cover it (Lobb et al., 2013).

Inability to understand the language is an extremely important barrier faced by migrants, as it leads to inability to communicate, frustration and ultimately abandonment of efforts to use health services (Ahmad et al., 2012; Sun et al., 2010; Todd et al., 2011; Woloshin et al., 1997). Language difficulties reduce the ability of migrants to communicate with healthcare staff and understand their instructions. In addition, the ability of migrants to inform themselves and increase their knowledge, such as the ability to browse websites related to health services or screening, is also significantly limited. Unfortunately, an important obstacle related to the health care system also contributes to this, which is the lack of translators who could improve the quality of communication between migrants and medical staff. 
medRxiv preprint doi: https://doi.org/10.1101/2022.02.24.22271449; this version posted March 2, 2022. The copyright holder for this preprint

The lack of a family doctor is another important barrier related to the health system, especially in the case of screening, such as in the case of mammography (Ahmad et al., 2012; Meana et al., 2001; Steven et al., 2004; Sun et al., 2010; Todd et al., 2011; Vahabi et al., 2016). Moreover, in some cases, the increased workload of doctors does not allow them to spend more time with migrants to explain more to them and answer their questions and queries especially in the case of screening. In fact, the lack of translators further exacerbates this problem, as doctors are unable to communicate with migrants, with the result that doctors' instructions and recommendations are not understood by migrants.

Particular reference needs to be made to the cultural barriers that migrants face in using health services. A typical example is the case of migrant women with particular religious beliefs. In this case, the existence of male doctors is a particular inhibiting factor for the use of health services related to sensitive issues, such as mammography (Ahmad et al., 2012; Steven et al., 2004; Todd et al., 2011; Vahabi et al., 2016). Some religions recognize women's bodies as 'sacred' and 'private', with the result that women may feel shame and guilt when a male doctor has to look at their bodies in the case of, for example, mammography. Many women, moreover, believe that the occurrence of a disease is God's will or the result of fate, so they do not use screening methods as they consider them unnecessary. In even more extreme situations, some societies consider breast cancer to be the result of immorality, so they view mammography negatively. The problem is further exacerbated by the fact that in some cases medical staff avoid discussing sensitive issues with migrants, in order to respect their cultural sensitivity and avoid possible friction and misunderstandings. This problem is extremely important, as in many cases the recommendation from health professionals is the most important motivation for migrants to undergo screening (Hanson et al., 2009). It is a fact that in most countries not much attention is paid to the cultural and religious specificities of migrants, which, especially in the case of the use of health services, are a decisive factor (Lobb et al., 2013).

Lack of knowledge is a key barrier to the use of health services by migrants, particularly in the case of screening. For example, migrant women are largely unaware of the risk factors for breast cancer (Ahmad et al., 2012; Meana et al., 2001; Vahabi et al., 
medRxiv preprint doi: https://doi.org/10.1101/2022.02.24.22271449; this version posted March 2, 2022. The copyright holder for this preprint (which was not certified by peer review) is the author/funder, who has granted medRxiv a license to display the preprint in perpetuity. It is made available under a CC-BY-NC-ND 4.0 International license .

2016). It is common, moreover, for migrant women to seek medical help only if they develop symptoms and signs, as health systems in their countries mainly emphasize treatment rather than prevention (Lobb et al., 2013). Similarly, many migrant women have limited knowledge about the benefits, side effects and the internetAsia of mammography and other screening methods. Thus, the lack of knowledge leads migrants to not use screening services as they consider them unnecessary (Hyman et al., 2001). The problem is magnified in the case of migrants who self-assess their health status as good, so that they consider that screening is not necessary (Garcia-Subirats et al., 2014; Jiménez-Rubio \& Hernández-Quevedo, 2011). Moreover, the lack of knowledge about how to access and navigate the health system creates even more problems, as migrants do not even know how to access the different services. It should be noted that the lack of knowledge is often combined with a lack of understanding of the language and a lack of translators in health services (Lobb et al., 2013).

Many migrants do not undergo screening because they are concerned about the consequences of being diagnosed with cancer, such as stress, access to necessary services, social isolation, poor prognosis, etc. (Ahmad et al., 2012; Vahabi et al., 2016). In addition, due to lack of knowledge, migrants are also concerned about the adverse effects of different tests, such as exposure to radiation during a mammogram, adverse effects of a therapeutic intervention (e.g. surgical removal of a tumor), etc. (Ahmad et al., 2012).

In conclusion, intensive efforts are needed to increase migrants' knowledge, implement culturally sensitive interventions in migrant communities and better inform health professionals so that they can reach migrants more effectively. Migrants are now a significant part of the population in host countries and appropriate conditions should be created for their best possible adaptation in order to safeguard and promote both migrant and public health. 
medRxiv preprint doi: https://doi.org/10.1101/2022.02.24.22271449; this version posted March 2, 2022. The copyright holder for this preprint (which was not certified by peer review) is the author/funder, who has granted medRxiv a license to display the preprint in perpetuity.

\section{It is made available under a CC-BY-NC-ND 4.0 International license .}
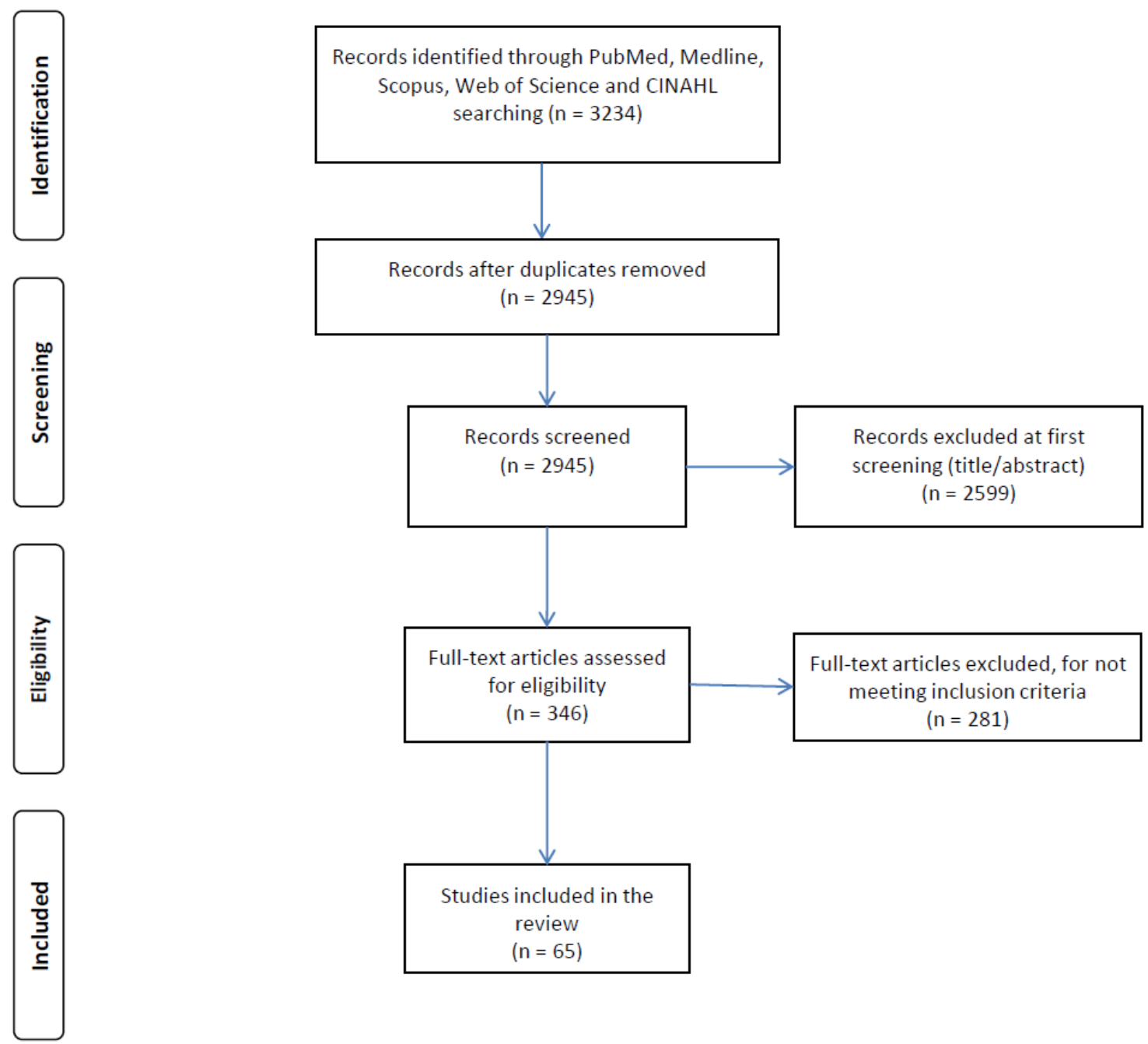

Figure 1. Flowchart of the systematic review. 
Table 1. Studies on migrants' access to health services, use of health services and the relative barriers.

\begin{tabular}{|c|c|c|c|c|c|c|c|c|c|}
\hline Reference & Country & Study year & Study design & $\begin{array}{l}\text { Participants } \\
\text { (n) }\end{array}$ & $\begin{array}{c}\text { Continent/country of } \\
\text { migrants }\end{array}$ & Data collection tools & $\begin{array}{l}\text { Comparison } \\
\text { with natives }\end{array}$ & $\begin{array}{c}\text { Access and use of } \\
\text { healthcare services }\end{array}$ & Barriers \\
\hline (X. Liu et al., 2017) & $\begin{array}{l}\text { United } \\
\text { Kingdom }\end{array}$ & 2017 & Qualitative & 54 & China & $\begin{array}{c}\text { Focus groups and } \\
\text { interviews }\end{array}$ & & $\downarrow$ & $\uparrow$ \\
\hline (Ellins \& Glasby, 2016) & $\begin{array}{c}\text { United } \\
\text { Kingdom }\end{array}$ & 2012 & Qualitative & 20 & Asia & Interviews & & $\downarrow$ & 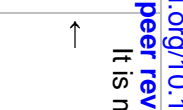 \\
\hline (Suurmond et al., 2016) & Netherlands & 2012 & Qualitative & 55 & $\begin{array}{l}\text { Turkey, Morocco, } \\
\text { Surinam }\end{array}$ & Interviews & & $\downarrow$ & 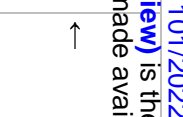 \\
\hline (Saltus \& Pithara, 2015) & Wales & 2013 & Qualitative & 32 & Asia & Interviews & & $\downarrow$ & $\uparrow$ \\
\hline (Thyli et al., 2014) & Norway & 2009 & Qualitative & 15 & $\begin{array}{c}\text { Iran, Iraq, Bosnia- } \\
\text { Herzegovina, Vietnam }\end{array}$ & Interviews & & $\downarrow$ & 돈 \\
\hline (Z. Liu et al., 2015) & $\begin{array}{l}\text { United } \\
\text { Kingdom }\end{array}$ & 2015 & Qualitative & 33 & China & Interviews & & $\downarrow$ & 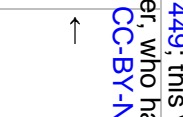 \\
\hline (Doshani et al., 2007) & $\begin{array}{c}\text { United } \\
\text { Kingdom }\end{array}$ & 2007 & Qualitative & 24 & Asia & Focus groups & & $\downarrow$ & 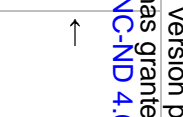 \\
\hline (Nielsen et al., 2012) & Denmark & 2007 & Cross-sectional & 4.952 & $\begin{array}{l}\text { Serbia, Croatia, Iran, } \\
\text { Iraq, Lebanon, Pakistan, } \\
\text { Somali, Turkey }\end{array}$ & Questionnaire & $\mathrm{X}$ & $\begin{array}{c}\uparrow \text { family physician } \\
\uparrow \text { hospitalization } \\
\uparrow \text { outpatients clinics } \\
\uparrow \text { ER } \\
\downarrow \text { dentist }\end{array}$ & 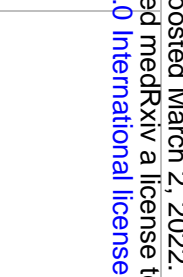 \\
\hline (Buja et al., 2014) & Italy & 2013 & Cross-sectional & 35,541 & $\begin{array}{l}\text { Europe, Asia, Africa, } \\
\text { Latin America, Oceania }\end{array}$ & Questionnaire & $\mathrm{X}$ & $\uparrow \mathrm{ER}$ & \\
\hline (Berens et al., 2014) & Germany & $2010-2011$ & Cross-sectional & 208,500 & Turkey & Files & $\mathrm{X}$ & $\uparrow$ screening & \\
\hline (Garcia-Subirats et al., 2014) & Spain & 2006-2012 & Cross-sectional & 31,063 & $\begin{array}{c}\text { Europe, Asia, Africa, } \\
\text { Latin America }\end{array}$ & Questionnaire & $\mathrm{X}$ & $\uparrow$ family physician & \\
\hline (Ricardo-Rodrigues et al., & Spain & 2011 & Cross-sectional & 5,303 & & Questionnaire & $\mathrm{X}$ & $\downarrow$ screening & \\
\hline
\end{tabular}




\begin{tabular}{|c|c|c|c|c|c|c|c|c|c|}
\hline 2015) & & & & & & & & & \\
\hline (Solé-Auró et al., 2012) & $\begin{array}{c}\text { Austria, } \\
\text { Belgium, } \\
\text { Denmark, } \\
\text { France, } \\
\text { Germany, } \\
\text { Greece, Italy, } \\
\text { Netherlands, } \\
\text { Spain, } \\
\text { Sweden, } \\
\text { Switzerland }\end{array}$ & 2004 & Cross-sectional & 27,395 & $\begin{array}{c}\text { Europe, Asia, Africa, } \\
\text { Latin America }\end{array}$ & Questionnaire & $\mathrm{X}$ & $\uparrow$ family physician & 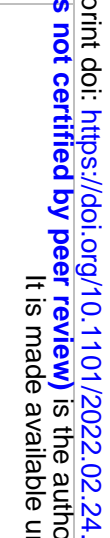 \\
\hline (Glaesmer et al., 2011) & Germany & 2011 & Cross-sectional & 2,510 & $\begin{array}{c}\text { Europe, former Soviet } \\
\text { Union, Turkey }\end{array}$ & Questionnaire & $\mathrm{X}$ & $\begin{array}{c}\uparrow \text { family physician } \\
\uparrow \text { hospitalization } \\
\downarrow \text { outpatients clinics }\end{array}$ & 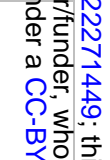 \\
\hline (Muñoz et al., 2012) & Spain & 2008 & Cross-sectional & 63,257 & $\begin{array}{c}\text { Europe, Asia, Africa, } \\
\text { Latin America }\end{array}$ & Files & $\mathrm{X}$ & $\begin{array}{l}\uparrow \text { family physician } \\
\uparrow \text { outpatients clinics }\end{array}$ & 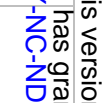 \\
\hline (Diaz et al., 2014) & Norway & 2008 & Cross-sectional & $2,977,933$ & & Files & $\mathrm{X}$ & $\uparrow$ family physician & 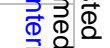 \\
\hline (Denktaş et al., 2009) & Netherlands & 2003 & Cross-sectional & 1503 & $\begin{array}{l}\text { Turkey, Morocco, } \\
\text { Surinam }\end{array}$ & Questionnaire & $\mathrm{X}$ & $\uparrow$ family physician & 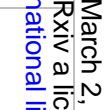 \\
\hline $\begin{array}{c}\text { (Hernández-Quevedo \& } \\
\text { Jiménez-Rubio, 2009) }\end{array}$ & Spain & 2003-2006 & Cross-sectional & 51,086 & $\begin{array}{l}\text { Europe, Asia, Africa, } \\
\text { Latin America, Oceania }\end{array}$ & Questionnaire & $\mathrm{X}$ & $\begin{array}{c}\uparrow \text { hospitalization } \\
\uparrow \text { ER } \\
\downarrow \text { outpatients clinics } \\
\downarrow \text { family physician }\end{array}$ & 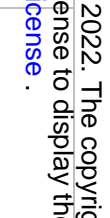 \\
\hline (Malmusi et al., 2014) & $\begin{array}{c}\text { Czech } \\
\text { Republic }\end{array}$ & 2008 & Cross-sectional & 1,955 & Ukraine & Questionnaire & $\mathrm{X}$ & $\begin{array}{c}\downarrow \text { outpatients clinics } \\
\downarrow \text { family physician } \\
\downarrow \text { ER }\end{array}$ & 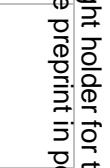 \\
\hline
\end{tabular}




\begin{tabular}{|c|c|c|c|c|c|c|c|c|c|}
\hline & & & & & & & & $\downarrow$ dentist & $\sum_{0} \frac{\mathrm{O}}{\Phi}$ \\
\hline $\begin{array}{c}\text { (Jiménez-Rubio \& } \\
\text { Hernández-Quevedo, 2011) }\end{array}$ & Spain & $2003-2006$ & Cross-sectional & 51,086 & $\begin{array}{c}\text { Europe, Asia, Africa, } \\
\text { Latin America, Oceania }\end{array}$ & Questionnaire & $\mathrm{X}$ & $\begin{array}{c}\uparrow \mathrm{ER} \\
\downarrow \text { family physician } \\
\downarrow \text { outpatients clinics }\end{array}$ & 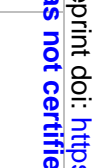 \\
\hline (de Back et al., 2015) & Netherlands & 2009-2010 & Cross-sectional & 58,274 & Antilles & Files & $\mathrm{X}$ & $\downarrow$ family physician & \\
\hline (Gimeno-Feliu et al., 2013) & Spain & 2007 & Cross-sectional & 594,145 & $\begin{array}{l}\text { Europe, Asia, Africa, } \\
\text { Latin America, Oceania }\end{array}$ & Files & $\mathrm{X}$ & $\downarrow$ family physician & 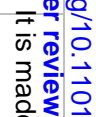 \\
\hline (De Luca et al., 2013) & Italy & $2004-2005$ & Cross-sectional & 102,857 & $\begin{array}{l}\text { Europe, Asia, Africa, } \\
\text { Latin America }\end{array}$ & Questionnaire & $\mathrm{X}$ & $\begin{array}{c}\uparrow \mathrm{ER} \\
\downarrow \text { outpatients clinics }\end{array}$ & 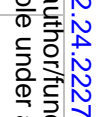 \\
\hline (Rinaldi et al., 2016) & Italy & 2011-2014 & Cross-sectional & 45,645 & $\begin{array}{c}\text { Asia, Africa, Ukraine, } \\
\text { Moldova, Servia, } \\
\text { Albania }\end{array}$ & Files & $\mathrm{X}$ & $\uparrow$ hospitalization & 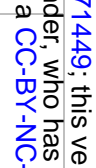 \\
\hline (de Bruijne et al., 2013) & Netherlands & $1995-2005$ & Cross-sectional & 433,501 & $\begin{array}{l}\text { Morocco, Turkey, } \\
\text { Surinam, Antilles }\end{array}$ & Files & $\mathrm{X}$ & $\uparrow$ hospitalization & 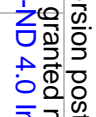 \\
\hline (Ramos et al., 2013) & Spain & 2011 & Cross-sectional & 42,839 & $\begin{array}{c}\text { Europe, Asia, Africa, } \\
\text { Latin America }\end{array}$ & Files & $\mathrm{X}$ & $\downarrow$ hospitalization & 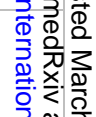 \\
\hline (Neergaard et al., 2013) & Denmark & 2006 & Cross-sectional & 599 & Europe & Files & $\mathrm{X}$ & $\uparrow$ outpatients clinics & \\
\hline (Ruud et al., 2015) & Norway & 2009 & Cross-sectional & 3,864 & $\begin{array}{l}\text { Sweden, Pakistan, } \\
\text { Somali, Poland }\end{array}$ & Questionnaire & $\mathrm{X}$ & $\uparrow \mathrm{ER}$ & \\
\hline $\begin{array}{c}\text { (Carrasco-Garrido et al., } \\
\text { 2009) }\end{array}$ & Spain & 2006 & Cross-sectional & 28,042 & $\begin{array}{l}\text { Europe, Asia, Africa, } \\
\text { Latin America }\end{array}$ & Questionnaire & $\mathrm{X}$ & $\uparrow \mathrm{ER}$ & \\
\hline (Sandvik et al., 2012) & Norway & 2008 & Cross-sectional & 715,278 & $\begin{array}{l}\text { Poland, Germany, Iraq, } \\
\text { Somali }\end{array}$ & Files & $\mathrm{X}$ & $\downarrow$ ER & \\
\hline (Jensen et al., 2012) & Denmark & 2008-2009 & Cross-sectional & 149,234 & Europe, Asia, Africa, & Files & $\mathrm{X}$ & $\downarrow$ screening & \\
\hline
\end{tabular}




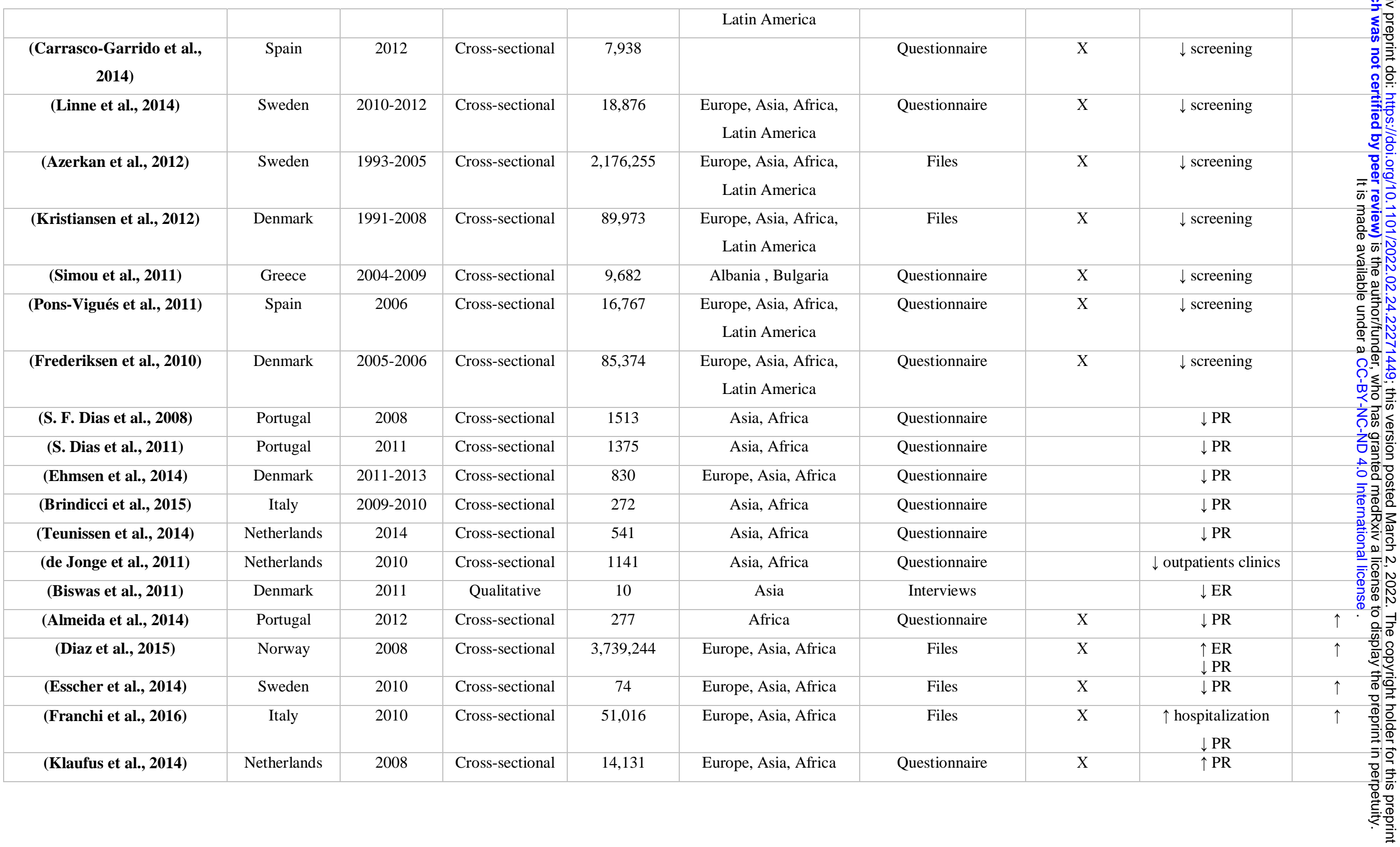




\begin{tabular}{|c|c|c|c|c|c|c|c|c|c|}
\hline (Koopmans et al., 2013) & Netherlands & $2001-2003$ & Cross-sectional & 9,077 & Europe, Asia, Africa & Questionnaire & $\mathrm{X}$ & $\downarrow \mathrm{PR}$ & $\uparrow$ \\
\hline (Nielsen et al., 2015) & Sweden & 2007 & Cross-sectional & 3,573 & Europe, Asia, Africa & Questionnaire & $\mathrm{X}$ & $\downarrow \mathrm{PR}$ & $\uparrow$ \\
\hline (Spinogatti et al., 2015) & Italy & $2001-2010$ & Cross-sectional & 139,775 & Europe, Asia, Africa & Files & $\mathrm{X}$ & $\begin{array}{l}\uparrow \mathrm{ER} \\
\downarrow \mathrm{PR}\end{array}$ & $\uparrow$ \\
\hline (Straiton et al., 2014) & Norway & 2008 & Cross-sectional & $2,712,974$ & Europe, Asia, Africa & Files & $\mathrm{X}$ & $\begin{array}{l}\downarrow \mathrm{ER} \\
\downarrow \mathrm{PR}\end{array}$ & $\uparrow$ \\
\hline (Villarroel \& Artazcoz, 2016) & Spain & 20 & Cross-sectional & 22,234 & Europe, Asia, Africa & Questionnaire & $\mathrm{X}$ & $\downarrow \mathrm{PR}$ & $\uparrow$ \\
\hline (Galanis et al., 2013) & Greece & 2012 & Cross-sectional & 191 & $\begin{array}{l}\text { Asia, Africa, former } \\
\text { Soviet Union, Balkan }\end{array}$ & Questionnaire & & $\downarrow$ & $\uparrow$ \\
\hline (Sourtzi et al., 2020) & Greece & 2013-2014 & Cross-sectional & 1152 & $\begin{array}{l}\text { Asia, Africa, former } \\
\text { Soviet Union, Balkan }\end{array}$ & Questionnaire & & $\downarrow \mathrm{PR}$ & $\uparrow$ \\
\hline (Tsitsakis et al., 2017) & Greece & $2005-2011$ & Cross-sectional & 14,034 & $\begin{array}{l}\text { Asia, Africa, former } \\
\text { Soviet Union, Balkan }\end{array}$ & Files & $\mathrm{X}$ & $\begin{array}{c}\uparrow \text { ER } \\
\downarrow \text { hospitalization }\end{array}$ & $\uparrow$ \\
\hline (Kaitelidou et al., 2020) & Greece & 2013-2014 & Cross-sectional & 1854 & $\begin{array}{l}\text { Asia, Africa, former } \\
\text { Soviet Union, Balkan }\end{array}$ & Questionnaire & $\mathrm{X}$ & $\downarrow$ & $\uparrow$ \\
\hline (Stathopoulou et al., 2018) & Greece & 2016 & Cross-sectional & 1006 & Albania (mainly) & Questionnaire & $\mathrm{X}$ & $\downarrow$ & $\uparrow$ \\
\hline $\begin{array}{c}\text { (Chantzaras \& Yfantopoulos, } \\
\text { 2018) }\end{array}$ & Greece & 2016 & Cross-sectional & 1332 & Albania (mainly) & Questionnaire & $\mathrm{X}$ & $\downarrow$ & $\uparrow$ \\
\hline (Boutziona et al., 2020) & Greece & 2014 & Cross-sectional & 167 & & Questionnaire & & $\downarrow$ & $\uparrow$ \\
\hline
\end{tabular}

PR: primary healthcare

ER: emergency department 
Table 2. Detailed description of the barriers encountered by migrants in accessing and using healthcare services.

\begin{tabular}{|c|c|c|c|c|c|c|}
\hline Reference & $\begin{array}{l}\text { Language and } \\
\text { communication } \\
\text { difficulties }\end{array}$ & $\begin{array}{c}\text { Lack of information and } \\
\text { knowledge }\end{array}$ & $\begin{array}{l}\text { Family support } \\
\text { failure }\end{array}$ & $\begin{array}{l}\text { Cultural and religion } \\
\text { issues }\end{array}$ & $\begin{array}{c}\text { Fear for } \\
\text { pain }\end{array}$ & $\begin{array}{l}\text { Difficulties to understand } \\
\text { physicians' guidelines }\end{array}$ \\
\hline (X. Liu et al., 2017) & $\mathrm{X}$ & $\mathrm{X}$ & $\mathrm{X}$ & $\mathrm{X}$ & & \\
\hline (Galanis et al., 2013) & $\mathrm{X}$ & & & & & \\
\hline (Boutziona et al., 2020) & $\mathrm{X}$ & & & $\mathrm{X}$ & & \\
\hline (Koopmans et al., 2013) & $\mathrm{X}$ & & $\mathrm{X}$ & & & \\
\hline (Nielsen et al., 2015) & $\mathrm{X}$ & & $\mathrm{x}$ & & & \\
\hline (Spinogatti et al., 2015) & & & $\mathrm{x}$ & & & \\
\hline (Straiton et al., 2014) & & & $\mathrm{X}$ & & & \\
\hline (Villarroel \& Artazcoz, 2016) & & & $\mathrm{X}$ & & & \\
\hline (Beiser \& Hou, 2014) & $\mathrm{X}$ & & $\mathrm{X}$ & & & \\
\hline (Diaz et al., 2015) & & & $\mathrm{X}$ & & & \\
\hline (Durbin et al., 2014) & $\mathrm{X}$ & & & & & \\
\hline (Esscher et al., 2014) & $\mathrm{X}$ & & & & & \\
\hline (Klaufus et al., 2014) & & & $\mathrm{X}$ & & & \\
\hline (Almeida et al., 2014) & & & $\mathrm{X}$ & & & \\
\hline (Ho et al., 2005) & & & $\mathrm{X}$ & & & \\
\hline (Ellins \& Glasby, 2016) & $\mathrm{X}$ & $\mathrm{X}$ & & & & \\
\hline (Suurmond et al., 2016) & $\mathrm{x}$ & $\mathrm{X}$ & $\mathrm{x}$ & & & \\
\hline (Thyli et al., 2014) & $\mathrm{X}$ & & $\mathrm{X}$ & & $\mathrm{X}$ & \\
\hline (Z. Liu et al., 2015) & $\mathrm{X}$ & & & & & $\mathrm{X}$ \\
\hline (Doshani et al., 2007) & & $\mathrm{X}$ & & & & \\
\hline (Garcia-Subirats et al., 2014) & & & & & $\mathrm{X}$ & \\
\hline (Denktaş et al., 2009) & $\mathrm{X}$ & & & & & \\
\hline
\end{tabular}


Table 2 (continued). Detailed description of the barriers encountered by migrants in accessing and using healthcare services.

\begin{tabular}{|c|c|c|c|c|c|c|c|}
\hline Паралоцли́ & $\begin{array}{l}\text { Females shame } \\
\text { and modesty }\end{array}$ & Long waiting hour & $\begin{array}{l}\text { Lack of } \\
\text { time }\end{array}$ & $\begin{array}{l}\text { High } \\
\text { cost }\end{array}$ & $\begin{array}{l}\text { Lack of } \\
\text { insurance }\end{array}$ & Emotional barriers & $\begin{array}{c}\text { Functional } \\
\text { disability }\end{array}$ \\
\hline (Galanis et al., 2013) & & $\mathrm{X}$ & & $\mathrm{X}$ & & $\mathrm{X}$ & \\
\hline (Boutziona et al., 2020) & & & & $\mathrm{X}$ & $\mathrm{X}$ & & \\
\hline (Almeida et al., 2014) & & & & & $\mathrm{X}$ & & \\
\hline (Doshani et al., 2007) & $\mathrm{X}$ & & & & $\mathrm{x}$ & & \\
\hline (Garcia-Subirats et al., 2014) & & $\mathrm{X}$ & $\mathrm{X}$ & $\mathrm{X}$ & $\mathrm{X}$ & & \\
\hline (Denktaş et al., 2009) & & & & & $\mathrm{X}$ & & \\
\hline (X. Liu et al., 2017) & & $\mathrm{X}$ & & & $\mathrm{x}$ & & \\
\hline (Sourtzi et al., 2020) & $\mathrm{X}$ & & & & $\mathrm{X}$ & & \\
\hline (Boutziona et al., 2020) & $\mathrm{X}$ & & & & $\mathrm{X}$ & & \\
\hline (Esscher et al., 2014) & $\mathrm{X}$ & & & & $\mathrm{X}$ & & \\
\hline (Klaufus et al., 2014) & $\mathrm{x}$ & & & & & & $\mathrm{X}$ \\
\hline (Nielsen et al., 2015) & $\mathrm{X}$ & & & & & & $\mathrm{X}$ \\
\hline (Spinogatti et al., 2015) & $\mathrm{X}$ & & & & & & \\
\hline (Villarroel \& Artazcoz, 2016) & $\mathrm{X}$ & & & & & & \\
\hline (Jiménez-Rubio \& Hernández-Quevedo, 2011) & $\mathrm{X}$ & & & & & & \\
\hline (Garcia-Subirats et al., 2014) & $\mathrm{X}$ & & & & & & \\
\hline (Suurmond et al., 2016) & & & $\mathrm{X}$ & & & & \\
\hline (Denktaş et al., 2009) & & & $\mathrm{X}$ & & & & \\
\hline
\end{tabular}


Table 2 (continued). Detailed description of the barriers encountered by migrants in accessing and using healthcare services.

\begin{tabular}{|c|c|c|c|c|c|c|c|}
\hline Паралорли́ & $\begin{array}{c}\text { Short length of } \\
\text { stay }\end{array}$ & Low income & $\begin{array}{l}\text { Stay in rural } \\
\text { areas }\end{array}$ & $\begin{array}{c}\text { Low educational } \\
\text { level }\end{array}$ & $\begin{array}{c}\text { Limited social } \\
\text { integration }\end{array}$ & Lack of trust & $\begin{array}{l}\text { Self-esteem of good } \\
\text { health status }\end{array}$ \\
\hline (Saltus \& Pithara, 2015) & & & & & $\mathrm{X}$ & & \\
\hline (Spinogatti et al., 2015) & & & & $\mathrm{X}$ & & & \\
\hline (Straiton et al., 2014) & $\mathrm{X}$ & $\mathrm{X}$ & & & & & \\
\hline (Villarroel \& Artazcoz, 2016) & & & & $\mathrm{X}$ & & & \\
\hline (Sourtzi et al., 2020) & & $\mathrm{X}$ & & & & & \\
\hline (Beiser \& Hou, 2014) & $\mathrm{x}$ & & & & & & \\
\hline (Diaz et al., 2015) & & $\mathrm{X}$ & $\mathrm{X}$ & $\mathrm{X}$ & & & \\
\hline (Durbin et al., 2014) & & $\mathrm{X}$ & & $\mathrm{X}$ & & & \\
\hline (Esscher et al., 2014) & & $\mathrm{X}$ & & $\mathrm{x}$ & & & \\
\hline (Klaufus et al., 2014) & & $\mathrm{X}$ & & $\mathrm{x}$ & $\mathrm{X}$ & & \\
\hline (Koopmans et al., 2013) & & & & $\mathrm{X}$ & & & \\
\hline (Nielsen et al., 2015) & $\mathrm{x}$ & $\mathrm{X}$ & & & & & \\
\hline (Almeida et al., 2014) & & $\mathrm{X}$ & & $\mathrm{X}$ & & & \\
\hline (Ricardo-Rodrigues et al., 2015) & & & $\mathrm{X}$ & $\mathrm{X}$ & & & \\
\hline (Solé-Auró et al., 2012) & & & & & & & \\
\hline (Diaz et al., 2014) & $\mathrm{X}$ & $\mathrm{x}$ & & & & & \\
\hline (Jiménez-Rubio \& Hernández-Quevedo, 2011) & & $\mathrm{X}$ & & & & & \\
\hline (Diaz \& Kumar, 2014) & $\mathrm{X}$ & & & & & & \\
\hline (Kristiansen et al., 2012) & & & & $\mathrm{X}$ & & & \\
\hline (Pons-Vigués et al., 2011) & & $\mathrm{X}$ & & & & & \\
\hline (Jiménez-Rubio \& Hernández-Quevedo, 2011) & & & & & & & $\mathrm{X}$ \\
\hline (Garcia-Subirats et al., 2014) & & & & & & & $\mathrm{x}$ \\
\hline (Thyli et al., 2014) & & & & & & $\mathrm{X}$ & \\
\hline
\end{tabular}


medRxiv preprint doi: https://doi.org/10.1101/2022.02.24.22271449; this version posted March 2, 2022. The copyright holder for this preprint (which was not certified by peer review) is the author/funder, who has granted medRxiv a license to display the preprint in perpetuity.

\section{References}

Abe-Kim, J., Takeuchi, D. T., Hong, S., Zane, N., Sue, S., Spencer, M. S., Appel, H., Nicdao, E., \& Alegría, M. (2007). Use of mental health-related services among immigrant and US-born Asian Americans: Results from the National Latino and Asian American Study. American Journal of Public Health, 97(1), 91-98. https://doi.org/10.2105/AJPH.2006.098541

Ahmad, F., Mahmood, S., Pietkiewicz, I., McDonald, L., \& Ginsburg, O. (2012). Concept mapping with South Asian immigrant women: Barriers to mammography and solutions. Journal of Immigrant and Minority Health, 14(2), 242-250. https://doi.org/10.1007/s10903-011-9472-7

Alegría, M., Cao, Z., McGuire, T. G., Ojeda, V. D., Sribney, B., Woo, M., \& Takeuchi, D. (2006). Health insurance coverage for vulnerable populations: Contrasting Asian Americans and Latinos in the United States. Inquiry: A Journal of Medical Care Organization, Provision and Financing, 43(3), 231-254. https://doi.org/10.5034/inquiryjrnl_43.3.231

Almeida, L. M., Santos, C. C., Caldas, J. P., Ayres-de-Campos, D., \& Dias, S. (2014). Obstetric care in a migrant population with free access to health care. International Journal of Gynaecology and Obstetrics: The Official Organ of the International Federation of Gynaecology and Obstetrics, 126(3), 244-247. https://doi.org/10.1016/j.ijgo.2014.03.023

Azerkan, F., Sparén, P., Sandin, S., Tillgren, P., Faxelid, E., \& Zendehdel, K. (2012). Cervical screening participation and risk among Swedish-born and immigrant women in Sweden. International Journal of Cancer, 130(4), 937-947. https://doi.org/10.1002/ijc.26084

Beiser, M., \& Hou, F. (2014). Chronic health conditions, labour market participation and resource consumption among immigrant and native-born residents of Canada. International Journal of Public Health, 59(3), 541-547. https://doi.org/10.1007/s00038-014-0544-z

Berens, E.-M., Stahl, L., Yilmaz-Aslan, Y., Sauzet, O., Spallek, J., \& Razum, O. (2014). Participation in breast cancer screening among women of Turkish origin in 
medRxiv preprint doi: https://doi.org/10.1101/2022.02.24.22271449; this version posted March 2, 2022. The copyright holder for this preprint (which was not certified by peer review) is the author/funder, who has granted medRxiv a license to display the preprint in perpetuity. It is made available under a CC-BY-NC-ND 4.0 International license .

Germany - a register-based study. BMC Women's Health, 14(1), 24. https://doi.org/10.1186/1472-6874-14-24

Biswas, D., Kristiansen, M., Krasnik, A., \& Norredam, M. (2011). Access to healthcare and alternative health-seeking strategies among undocumented migrants in Denmark. BMC Public Health, 11, 560. https://doi.org/10.1186/1471-2458-11560

Boutziona, I., Papanikolaou, D., Sokolakis, I., Mytilekas, K. V., \& Apostolidis, A. (2020). Healthcare Access, Quality, and Satisfaction Among Albanian Immigrants Using the Emergency Department in Northern Greece. Journal of Immigrant and Minority Health, 22(3), 512-525. https://doi.org/10.1007/s10903-020-00983-X

Brindicci, G., Trillo, G., Santoro, C. R., Volpe, A., Monno, L., \& Angarano, G. (2015). Access to health services for undocumented immigrants in Apulia. Journal of Immigrant and Minority Health, 17(2), 618-623. https://doi.org/10.1007/s10903014-0009-8

Buja, A., Fusco, M., Furlan, P., Bertoncello, C., Baldovin, T., Casale, P., Marcolongo, A., \& Baldo, V. (2014). Characteristics, processes, management and outcome of accesses to accident and emergency departments by citizenship. International Journal of Public Health, 59(1), 167-174. https://doi.org/10.1007/s00038-0130483-0

Callahan, S. T., Hickson, G. B., \& Cooper, W. O. (2006). Health care access of Hispanic young adults in the United States. The Journal of Adolescent Health: Official Publication of the Society for Adolescent Medicine, 39(5), 627-633. https://doi.org/10.1016/j.jadohealth.2006.04.012

Carrasco-Garrido, P., Hernandez-Barrera, V., Lopez de Andres, A., Jimenez-Trujillo, I., Gallardo Pino, C., \& Jimenez-Garcia, R. (2014). Awareness and uptake of colorectal, breast, cervical and prostate cancer screening tests in Spain. European Journal of Public Health, 24(2), 264-270. https://doi.org/10.1093/eurpub/ckt089

Carrasco-Garrido, P., Jiménez-García, R., Barrera, V. H., de Andrés, A. L., \& de Miguel, A. G. (2009). Significant differences in the use of healthcare resources of nativeborn and foreign born in Spain. BMC Public Health, 9, 201. https://doi.org/10.1186/1471-2458-9-201 
medRxiv preprint doi: https://doi.org/10.1101/2022.02.24.22271449; this version posted March 2, 2022. The copyright holder for this preprint (which was not certified by peer review) is the author/funder, who has granted medRxiv a license to display the preprint in perpetuity. It is made available under a CC-BY-NC-ND 4.0 International license .

Chantzaras, A. E., \& Yfantopoulos, J. N. (2018). Income-related health inequalities among the migrant and native-born populations in Greece during the economic crisis: A decomposition analysis. European Journal of Public Health, 28(supp1_5), 24-31. https://doi.org/10.1093/eurpub/cky203

Cunningham, P. J. (2006). What accounts for differences in the use of hospital emergency departments across U.S. communities? Health Affairs (Project Hope), 25(5), w324-336. https://doi.org/10.1377/hlthaff.25.w324

de Back, T. R., Bodewes, A. J., Brewster, L. M., \& Kunst, A. E. (2015). Cardiovascular Health and Related Health Care Use of Moluccan-Dutch Immigrants. PloS One, 10(9), e0138644. https://doi.org/10.1371/journal.pone.0138644

de Bruijne, M. C., van Rosse, F., Uiters, E., Droomers, M., Suurmond, J., Stronks, K., \& Essink-Bot, M.-L. (2013). Ethnic variations in unplanned readmissions and excess length of hospital stay: A nationwide record-linked cohort study. European Journal of Public Health, 23(6), 964-971. https://doi.org/10.1093/eurpub/ckt005

de Jonge, A., Rijnders, M., Agyemang, C., van der Stouwe, R., den Otter, J., Van den Muijsenbergh, M. E. T. C., \& Buitendijk, S. (2011). Limited midwifery care for undocumented women in the Netherlands. Journal of Psychosomatic Obstetrics and Gynaecology, 32(4), 182-188. https://doi.org/10.3109/0167482X.2011.589016

De Luca, G., Ponzo, M., \& Andrés, A. R. (2013). Health care utilization by immigrants in Italy. International Journal of Health Care Finance and Economics, 13(1), 1-31. https://doi.org/10.1007/s10754-012-9119-9

Denktaş, S., Koopmans, G., Birnie, E., Foets, M., \& Bonsel, G. (2009). Ethnic background and differences in health care use: A national cross-sectional study of native Dutch and immigrant elderly in the Netherlands. International Journal for Equity in Health, 8(1), 35. https://doi.org/10.1186/1475-9276-8-35

Derose, K. P., Bahney, B. W., Lurie, N., \& Escarce, J. J. (2009). Review: Immigrants and health care access, quality, and cost. Medical Care Research and Review: MCRR, 66(4), 355-408. https://doi.org/10.1177/1077558708330425 
medRxiv preprint doi: https://doi.org/10.1101/2022.02.24.22271449; this version posted March 2, 2022. The copyright holder for this preprint (which was not certified by peer review) is the author/funder, who has granted medRxiv a license to display the preprint in perpetuity. It is made available under a CC-BY-NC-ND 4.0 International license .

Dias, S. F., Severo, M., \& Barros, H. (2008). Determinants of health care utilization by immigrants in Portugal. BMC Health Services Research, 8(1), 207. https://doi.org/10.1186/1472-6963-8-207

Dias, S., Gama, A., Cortes, M., \& de Sousa, B. (2011). Healthcare-seeking patterns among immigrants in Portugal. Health \& Social Care in the Community, 19(5), 514-521. https://doi.org/10.1111/j.1365-2524.2011.00996.X

Diaz, E., Calderón-Larrañaga, A., Prado-Torres, A., Poblador-Plou, B., \& Gimeno-Feliu, L.-A. (2015). How do immigrants use primary health care services? A registerbased study in Norway. European Journal of Public Health, 25(1), 72-78. https://doi.org/10.1093/eurpub/cku123

Diaz, E., Gimeno-Feliu, L.-A., Calderón-Larrañaga, A., \& Prados-Torres, A. (2014). Frequent attenders in general practice and immigrant status in Norway: A nationwide cross-sectional study. Scandinavian Journal of Primary Health Care, 32(4), 232-240. https://doi.org/10.3109/02813432.2014.982368

Diaz, E., \& Kumar, B. N. (2014). Differential utilization of primary health care services among older immigrants and Norwegians: A register-based comparative study in Norway. BMC Health Services Research, 14, 623. https://doi.org/10.1186/s12913014-0623-0

Donnelly, T. T., McKellin, W., Hislop, G., \& Long, B. (2009). Socioeconomic influences on Vietnamese-Canadian women's breast and cervical cancer prevention practices: A social determinant's perspective. Social Work in Public Health, 24(5), 454-476. https://doi.org/10.1080/19371910802678772

Doshani, A., Pitchforth, E., Mayne, C. J., \& Tincello, D. G. (2007). Culturally sensitive continence care: A qualitative study among South Asian Indian women in Leicester. Family $\quad$ Practice, 24(6), 585-593. https://doi.org/10.1093/fampra/cmm058

Durbin, A., Lin, E., Moineddin, R., Steele, L. S., \& Glazier, R. H. (2014). Use of mental health care for nonpsychotic conditions by immigrants in different admission classes and by refugees in Ontario, Canada. Open Medicine: A Peer-Reviewed, Independent, Open-Access Journal, 8(4), e136-146. 
medRxiv preprint doi: https://doi.org/10.1101/2022.02.24.22271449; this version posted March 2, 2022. The copyright holder for this preprint (which was not certified by peer review) is the author/funder, who has granted medRxiv a license to display the preprint in perpetuity. It is made available under a CC-BY-NC-ND 4.0 International license .

Ehmsen, B. K., Biswas, D., Jensen, N. K., Krasnik, A., \& Norredam, M. (2014). Undocumented migrants have diverse health problems. Danish Medical Journal, 61(9), A4897.

Ellins, J., \& Glasby, J. (2016). "You don't know what you are saying 'Yes' and what you are saying 'No' to": Hospital experiences of older people from minority ethnic communities. Ageing and Society, 36(1), 42-63. https://doi.org/10.1017/S0144686X14000919

Esscher, A., Binder-Finnema, P., Bødker, B., Högberg, U., Mulic-Lutvica, A., \& Essén, B. (2014). Suboptimal care and maternal mortality among foreign-born women in Sweden: Maternal death audit with application of the "migration three delays" model. BMC Pregnancy and Childbirth, 14, 141. https://doi.org/10.1186/14712393-14-141

Franchi, C., Baviera, M., Sequi, M., Cortesi, L., Tettamanti, M., Roncaglioni, M. C., Pasina, L., Dignefa, C. D., Fortino, I., Bortolotti, A., Merlino, L., Mannucci, P. M., \& Nobili, A. (2016). Comparison of Health Care Resource Utilization by Immigrants Versus Native Elderly People. Journal of Immigrant and Minority Health, 18(1), 1-7. https://doi.org/10.1007/s10903-014-0152-2

Frederiksen, B. L., Jørgensen, T., Brasso, K., Holten, I., \& Osler, M. (2010). Socioeconomic position and participation in colorectal cancer screening. British Journal of Cancer, 103(10), 1496-1501. https://doi.org/10.1038/sj.bjc.6605962

Galanis, P., Sourtzi, P., Bellali, T., Theodorou, M., Karamitri, I., Siskou, O., Charalambous, G., \& Kaitelidou, D. (2013). Public health services knowledge and utilization among immigrants in Greece: A cross-sectional study. BMC Health Services Research, 13, 350. https://doi.org/10.1186/1472-6963-13-350

Garcia-Subirats, I., Vargas, I., Sanz-Barbero, B., Malmusi, D., Ronda, E., Ballesta, M., \& Vázquez, M. (2014). Changes in Access to Health Services of the Immigrant and Native-Born Population in Spain in the Context of Economic Crisis. International Journal of Environmental Research and Public Health, 11(10), 10182-10201. https://doi.org/10.3390/ijerph111010182

Gimeno-Feliu, L. A., Magallón-Botaya, R., Macipe-Costa, R. M., Luzón-Oliver, L., Cañada-Millan, J. L., \& Lasheras-Barrio, M. (2013). Differences in the use of 
medRxiv preprint doi: https://doi.org/10.1101/2022.02.24.22271449; this version posted March 2, 2022. The copyright holder for this preprint (which was not certified by peer review) is the author/funder, who has granted medRxiv a license to display the preprint in perpetuity. It is made available under a CC-BY-NC-ND 4.0 International license .

primary care services between Spanish national and immigrant patients. Journal of Immigrant and Minority Health, 15(3), 584-590. https://doi.org/10.1007/s10903-012-9647-X

Glaesmer, H., Wittig, U., Braehler, E., Martin, A., Mewes, R., \& Rief, W. (2011). Health care utilization among first and second generation immigrants and native-born Germans: A population-based study in Germany. International Journal of Public Health, 56(5), 541-548. https://doi.org/10.1007/s00038-010-0205-9

Guendelman, S., Schauffler, H. H., \& Pearl, M. (2001). Unfriendly shores: How immigrant children fare in the U.S. health system. Health Affairs (Project Hope), 20(1), 257-266. https://doi.org/10.1377/hlthaff.20.1.257

Hanson, K., Montgomery, P., Bakker, D., \& Conlon, M. (2009). Factors influencing mammography participation in Canada: An integrative review of the literature. Current Oncology (Toronto, Ont.), 16(5), 65-75. https://doi.org/10.3747/co.v16i5.359

Hernández-Quevedo, C., \& Jiménez-Rubio, D. (2009). A comparison of the health status and health care utilization patterns between foreigners and the national population in Spain: New evidence from the Spanish National Health Survey. Social Science \& Medicine (1982), 69(3), 370-378. https://doi.org/10.1016/j.socscimed.2009.05.005

Ho, V., Yamal, J. M., Atkinson, E. N., Basen-Engquist, K., Tortolero-Luna, G., \& Follen, M. (2005). Predictors of breast and cervical screening in Vietnamese women in Harris County, Houston, Texas. Cancer Nursing, 28(2), 119-129; quiz 130-131. https://doi.org/10.1097/00002820-200503000-00005

Huang, Z. J., Yu, S. M., \& Ledsky, R. (2006). Health status and health service access and use among children in U.S. immigrant families. American Journal of Public Health, 96(4), 634-640. https://doi.org/10.2105/AJPH.2004.049791

Hyman, I., Singh, M., Ahmad, F., Austin, L., Meana, M., George, U., Wells, L. M., \& Stewart, D. (2001). The role of physicians in mammography referral for older Caribbean women in Canada. Medscape Women's Health, 6(5), 6.

Jackson, J. S., Neighbors, H. W., Torres, M., Martin, L. A., Williams, D. R., \& Baser, R. (2007). Use of mental health services and subjective satisfaction with treatment 
medRxiv preprint doi: https://doi.org/10.1101/2022.02.24.22271449; this version posted March 2, 2022. The copyright holder for this preprint (which was not certified by peer review) is the author/funder, who has granted medRxiv a license to display the preprint in perpetuity. It is made available under a CC-BY-NC-ND 4.0 International license .

among Black Caribbean immigrants: Results from the National Survey of American Life. American Journal of Public Health, 97(1), 60-67. https://doi.org/10.2105/AJPH.2006.088500

Javier, J. R., Wise, P. H., \& Mendoza, F. S. (2007). The relationship of immigrant status with access, utilization, and health status for children with asthma. Ambulatory Pediatrics: The Official Journal of the Ambulatory Pediatric Association, 7(6), 421-430. https://doi.org/10.1016/j.ambp.2007.06.004

Jensen, L. F., Pedersen, A. F., Andersen, B., \& Vedsted, P. (2012). Identifying specific non-attending groups in breast cancer screening-Population-based registry study of participation and socio-demography. BMC Cancer, 12, 518. https://doi.org/10.1186/1471-2407-12-518

Jiménez-Rubio, D., \& Hernández-Quevedo, C. (2011). Inequalities in the use of health services between immigrants and the native population in Spain: What is driving the differences? The European Journal of Health Economics: HEPAC: Health Economics in Prevention and Care, 12(1), 17-28. https://doi.org/10.1007/s10198010-0220-z

Kaitelidou, D., Galanis, P., Economou, C., Mladovsky, P., Siskou, O., \& Sourtzi, P. (2020). Inequalities Between Migrants and Non-Migrants in Accessing and Using Health Services in Greece During an Era of Economic Hardship. International Journal of Health Services: Planning, Administration, Evaluation, 50(4), 444 457. https://doi.org/10.1177/0020731420902604

Karl-Trummer, U., Novak-Zezula, S., \& Metzler, B. (2010). Access to health care for undocumented migrants in the EU: A first landscape of NowHereland. Eurohealth, 16(1), 13-16.

Klaufus, L. H., Fassaert, T. J. L., \& de Wit, M. A. S. (2014). Equity of access to mental health care for anxiety and depression among different ethnic groups in four large cities in the Netherlands. Social Psychiatry and Psychiatric Epidemiology, 49(7), 1139-1149. https://doi.org/10.1007/s00127-014-0837-9

Koopmans, G. T., Uiters, E., Devillé, W., \& Foets, M. (2013). The use of outpatient mental health care services of migrants vis-a-vis Dutch natives: Equal access? The 
medRxiv preprint doi: https://doi.org/10.1101/2022.02.24.22271449; this version posted March 2, 2022. The copyright holder for this preprint (which was not certified by peer review) is the author/funder, who has granted medRxiv a license to display the preprint in perpetuity. It is made available under a CC-BY-NC-ND 4.0 International license .

International Journal of Social Psychiatry, 59(4), 342-350. https://doi.org/10.1177/0020764012437129

Kristiansen, M., Thorsted, B. L., Krasnik, A., \& von Euler-Chelpin, M. (2012). Participation in mammography screening among migrants and non-migrants in Denmark. Acta Oncologica (Stockholm, Sweden), 51(1), 28-36. https://doi.org/10.3109/0284186X.2011.626447

Lasser, K. E., Himmelstein, D. U., \& Woolhandler, S. (2006). Access to care, health status, and health disparities in the United States and Canada: Results of a crossnational population-based survey. American Journal of Public Health, 96(7), 1300-1307. https://doi.org/10.2105/AJPH.2004.059402

Lassetter, J. H., \& Callister, L. C. (2009). The impact of migration on the health of voluntary migrants in western societies. Journal of Transcultural Nursing: Official Journal of the Transcultural Nursing Society, 20(1), 93-104. https://doi.org/10.1177/1043659608325841

Linne, A., Leander, K., Lindström, D., Törnberg, S., \& Hultgren, R. (2014). Reasons for non-participation in population-based abdominal aortic aneurysm screening. The British Journal of Surgery, 101(5), 481-487. https://doi.org/10.1002/bjs.9434

Liu, X., Cook, G., \& Cattan, M. (2017). Support networks for Chinese older immigrants accessing English health and social care services: The concept of Bridge People. Health \& Social Care in the Community, 25(2), 667-677. https://doi.org/10.1111/hsc.12357

Liu, Z., Beaver, K., \& Speed, S. (2015). Chinese Elders' views on their interactions in general practice: A Grounded Theory study. Ethnicity \& Health, 20(2), 129-144. https://doi.org/10.1080/13557858.2014.890176

Lobb, R., Pinto, A. D., \& Lofters, A. (2013). Using concept mapping in the knowledgeto-action process to compare stakeholder opinions on barriers to use of cancer screening among South Asians. Implementation Science, 8(1), 37. https://doi.org/10.1186/1748-5908-8-37

Loue, S., \& Sajatovic, M. (2012). Encyclopedia of immigrant health (Springer). 
medRxiv preprint doi: https://doi.org/10.1101/2022.02.24.22271449; this version posted March 2, 2022. The copyright holder for this preprint (which was not certified by peer review) is the author/funder, who has granted medRxiv a license to display the preprint in perpetuity. It is made available under a CC-BY-NC-ND 4.0 International license .

Macpherson, D. W., Gushulak, B. D., \& Macdonald, L. (2007). Health and foreign policy: Influences of migration and population mobility. Bulletin of the World Health Organization, 85(3), 200-206. https://doi.org/10.2471/blt.06.036962

Malmusi, D., Borrell, C., \& Benach, J. (2010). Migration-related health inequalities: Showing the complex interactions between gender, social class and place of origin. Social Science \& Medicine (1982), 71(9), 1610-1619. https://doi.org/10.1016/j.socscimed.2010.07.043

Malmusi, D., Drbohlav, D., Dzúrová, D., Palència, L., \& Borrell, C. (2014). Inequalities in healthcare access by type of visa in a context of restrictive health insurance policy: The case of Ukrainians in Czechia. International Journal of Public Health, 59(5), 715-719. https://doi.org/10.1007/s00038-014-0592-4

Martín-López, R., Jiménez-García, R., Lopez-de-Andres, A., Hernández-Barrera, V., Jiménez-Trujillo, I., Gil-de-Miguel, A., \& Carrasco-Garrido, P. (2013). Inequalities in uptake of breast cancer screening in Spain: Analysis of a crosssectional national survey. Public Health, 127(9), 822-827. https://doi.org/10.1016/j.puhe.2013.03.006

Meana, M., Bunston, T., George, U., Wells, L., \& Rosser, W. (2001). Influences on breast cancer screening behaviors in Tamil immigrant women 50 years old and over. Ethnicity \& Health, 6(3-4), 179-188. https://doi.org/10.1080/13557850120078107

Muñoz, M.-A., Pastor, E., Pujol, J., Del Val, J. L., Cordomí, S., \& Hermosilla, E. (2012). Primary health care utilization by immigrants as compared to the native population: A multilevel analysis of a large clinical database in Catalonia. European Journal of General Practice, 18(2), 100-106. https://doi.org/10.3109/13814788.2012.656085

Neergaard, M. A., Jensen, A. B., Olesen, F., \& Vedsted, P. (2013). Access to outreach specialist palliative care teams among cancer patients in Denmark. Journal of Palliative Medicine, 16(8), 951-957. https://doi.org/10.1089/jpm.2012.0265

Nielsen, S. S., Hempler, N. F., Waldorff, F. B., Kreiner, S., \& Krasnik, A. (2012). Is there equity in use of healthcare services among immigrants, their descendents, and 
medRxiv preprint doi: https://doi.org/10.1101/2022.02.24.22271449; this version posted March 2, 2022. The copyright holder for this preprint (which was not certified by peer review) is the author/funder, who has granted medRxiv a license to display the preprint in perpetuity. It is made available under a CC-BY-NC-ND 4.0 International license .

ethnic Danes? Scandinavian Journal of Public Health, 40(3), 260-270. https://doi.org/10.1177/1403494812443602

Nielsen, S. S., Jensen, N. K., Kreiner, S., Norredam, M., \& Krasnik, A. (2015). Utilisation of psychiatrists and psychologists in private practice among nonWestern labour immigrants, immigrants from refugee-generating countries and ethnic Danes: The role of mental health status. Social Psychiatry and Psychiatric Epidemiology, 50(1), 67-76. https://doi.org/10.1007/s00127-014-0916-y

Pons-Vigués, M., Puigpinós-Riera, R., Rodríguez-Sanz, M., Serral, G., Palència, L., \& Borrell, C. (2011). Preventive control of breast and cervical cancer in immigrant and native women in Spain: The role of country of origin and social class. International Journal of Health Services: Planning, Administration, Evaluation, 41(3), 483-499. https://doi.org/10.2190/HS.41.3.e

Ramos, J. M., Navarrete-Muñoz, E. M., Pinargote, H., Sastre, J., Seguí, J. M., \& Rugero, M. J. (2013). Hospital admissions in Alicante (Spain): A comparative analysis of foreign citizens from high-income countries, immigrants from low-income countries, and Spanish citizens. BMC Health Services Research, 13, 510. https://doi.org/10.1186/1472-6963-13-510

Ricardo-Rodrigues, I., Jiménez-García, R., Hernández-Barrera, V., Carrasco-Garrido, P., Jiménez-Trujillo, I., \& López de Andrés, A. (2015). Social disparities in access to breast and cervical cancer screening by women living in Spain. Public Health, 129(7), 881-888. https://doi.org/10.1016/j.puhe.2015.02.021

Rinaldi, F., Nembrini, S., Concoreggi, C., Magoni, M., \& Padovani, A. (2016). Neurological diseases and health care utilization among first-generation immigrants. Journal of Neurology, 263(4), 714-721. https://doi.org/10.1007/s00415-016-8029-Z

Ruud, S. E., Aga, R., Natvig, B., \& Hjortdahl, P. (2015). Use of emergency care services by immigrants-A survey of walk-in patients who attended the Oslo Accident and Emergency Outpatient Clinic. BMC Emergency Medicine, 15, 25. https://doi.org/10.1186/s12873-015-0055-0 
medRxiv preprint doi: https://doi.org/10.1101/2022.02.24.22271449; this version posted March 2, 2022. The copyright holder for this preprint (which was not certified by peer review) is the author/funder, who has granted medRxiv a license to display the preprint in perpetuity. It is made available under a CC-BY-NC-ND 4.0 International license .

Saltus, R., \& Pithara, C. (2015). "Care from the heart”: Older minoritised women's perceptions of dignity in care. International Journal of Migration, Health and Social Care, 11(1), 57-70. https://doi.org/10.1108/IJMHSC-06-2014-0025

Sandvik, H., Hunskaar, S., \& Diaz, E. (2012). Immigrants' use of emergency primary health care in Norway: A registry-based observational study. BMC Health Services Research, 12, 308. https://doi.org/10.1186/1472-6963-12-308

Sanz, B., Regidor, E., Galindo, S., Pascual, C., Lostao, L., Díaz, J. M., \& Sánchez, E. (2011). Pattern of health services use by immigrants from different regions of the world residing in Spain. International Journal of Public Health, 56(5), 567-576. https://doi.org/10.1007/s00038-011-0237-9

Simou, E., Foundoulakis, E., Kourlaba, G., \& Maniadakis, N. (2011). Factors associated with the use of preventive services by women in Greece. European Journal of Public Health, 21(4), 512-519. https://doi.org/10.1093/eurpub/ckq103

Solé-Auró, A., Guillén, M., \& Crimmins, E. M. (2012). Health care usage among immigrants and native-born elderly populations in eleven European countries: Results from SHARE. The European Journal of Health Economics, 13(6), 741754. https://doi.org/10.1007/s10198-011-0327-x

Sourtzi, P., Galanis, P., Konstantakopoulou, O., Siskou, O., \& Kaitelidou, D. (2020). Factors that influence the health status of immigrants living in Greece. AIMS Public Health, 7(2), 287-300. https://doi.org/10.3934/publichealth.2020024

Spallek, J., Zeeb, H., \& Razum, O. (2011). What do we have to know from migrants' past exposures to understand their health status? A life course approach. Emerging Themes in Epidemiology, 8(1), 6. https://doi.org/10.1186/1742-7622-8-6

Spinogatti, F., Civenti, G., Conti, V., \& Lora, A. (2015). Ethnic differences in the utilization of mental health services in Lombardy (Italy): An epidemiological analysis. Social Psychiatry and Psychiatric Epidemiology, 50(1), 59-65. https://doi.org/10.1007/s00127-014-0922-0

Stathopoulou, T., Stornes, P., Mouriki, A., Kostaki, A., Cavounidis, J., Avrami, L., McNamara, C. L., Rapp, C., \& Eikemo, T. A. (2018). Health inequalities among migrant and native-born populations in Greece in times of crisis: The MIGHEAL 
medRxiv preprint doi: https://doi.org/10.1101/2022.02.24.22271449; this version posted March 2, 2022. The copyright holder for this preprint (which was not certified by peer review) is the author/funder, who has granted medRxiv a license to display the preprint in perpetuity. It is made available under a CC-BY-NC-ND 4.0 International license.

study. European Journal of Public Health, 28(suppl_5), 5-19. https://doi.org/10.1093/eurpub/cky225

Steven, D., Fitch, M., Dhaliwal, H., Kirk-Gardner, R., Sevean, P., Jamieson, J., \& Woodbeck, H. (2004). Knowledge, attitudes, beliefs, and practices regarding breast and cervical cancer screening in selected ethnocultural groups in Northwestern Ontario. Oncology Nursing Forum, 31(2), 305-311. https://doi.org/10.1188/04.ONF.305-311

Straiton, M., Reneflot, A., \& Diaz, E. (2014). Immigrants' use of primary health care services for mental health problems. BMC Health Services Research, 14, 341. https://doi.org/10.1186/1472-6963-14-341

Sun, Z., Xiong, H., Kearney, A., Zhang, J., Liu, W., Huang, G., \& Wang, P. P. (2010). Breast cancer screening among Asian immigrant women in Canada. Cancer Epidemiology, 34(1), 73-78. https://doi.org/10.1016/j.canep.2009.12.001

Suurmond, J., Rosenmöller, D. L., el Mesbahi, H., Lamkaddem, M., \& Essink-Bot, M.-L. (2016). Barriers in access to home care services among ethnic minority and Dutch elderly - A qualitative study. International Journal of Nursing Studies, 54, 23-35. https://doi.org/10.1016/j.ijnurstu.2015.02.014

Teunissen, E., van den Bosch, L., van Bavel, E., van den Driessen Mareeuw, F., van den Muijsenbergh, M., van Weel-Baumgarten, E., \& van Weel, C. (2014). Mental health problems in undocumented and documented migrants: A survey study. Family Practice, 31(5), 571-577. https://doi.org/10.1093/fampra/cmu038

The Lancet Public Health. (2018). No public health without migrant health. The Lancet Public Health, 3(6), e259. https://doi.org/10.1016/S2468-2667(18)30101-4

Thyli, B., Hedelin, B., \& Athlin, E. (2014). Experiences of health and care when growing old in Norway-From the perspective of elderly immigrants with minority ethnic backgrounds. Clinical Nursing Studies, 2(3), p52. https://doi.org/10.5430/cns.v2n3p52

Todd, L., Harvey, E., \& Hoffman-Goetz, L. (2011). Predicting breast and colon cancer screening among English-as-a-second-language older Chinese immigrant women to Canada. Journal of Cancer Education: The Official Journal of the American 
medRxiv preprint doi: https://doi.org/10.1101/2022.02.24.22271449; this version posted March 2, 2022. The copyright holder for this preprint (which was not certified by peer review) is the author/funder, who has granted medRxiv a license to display the preprint in perpetuity. It is made available under a CC-BY-NC-ND 4.0 International license .

Association for Cancer Education, 26(1), 161-169. https://doi.org/10.1007/s13187-010-0141-7

Tsitsakis, C. A., Karasavvoglou, A., Tsaridis, E., Ramantani, G., Florou, G., Polychronidou, P., \& Stamatakis, S. (2017). Features of public healthcare services provided to migrant patients in the Eastern Macedonia and Thrace Region (Greece). Health Policy (Amsterdam, Netherlands), 121(3), 329-337. https://doi.org/10.1016/j.healthpol.2016.12.006

Ullmann, S. H., Goldman, N., \& Massey, D. S. (2011). Healthier before they migrate, less healthy when they return? The health of returned migrants in Mexico. Social Science \& Medicine (1982), 73(3), 421-428. https://doi.org/10.1016/j.socscimed.2011.05.037

Vahabi, M., Lofters, A., Kumar, M., \& Glazier, R. H. (2016). Breast cancer screening disparities among immigrant women by world region of origin: A populationbased study in Ontario, Canada. Cancer Medicine, 5(7), 1670-1686. https://doi.org/10.1002/cam4.700

Villarroel, N., \& Artazcoz, L. (2016). Different Patterns in Health Care Use Among Immigrants in Spain. Journal of Immigrant and Minority Health, 18(2), 318-329. https://doi.org/10.1007/s10903-015-0202-4

Woloshin, S., Schwartz, L. M., Katz, S. J., \& Welch, H. G. (1997). Is language a barrier to the use of preventive services? Journal of General Internal Medicine, 12(8), 472-477. https://doi.org/10.1046/j.1525-1497.1997.00085.x

World Health Organization. (2008). Health of migrants. World Health Organization.

Yu, S. M., Huang, Z. J., Schwalberg, R. H., \& Nyman, R. M. (2006). Parental English proficiency and children's health services access. American Journal of Public Health, 96(8), 1449-1455. https://doi.org/10.2105/AJPH.2005.069500 